\title{
Manifold enhancement of electron beam induced deposition rate at grazing incidence
}

\author{
Ilya Sychugov, Yoshiko Nakayama and Kazutaka Mitsuishi \\ Quantum Dot Research Center, National Institute for Materials Science, Sakura 3-13,
}

Tsukuba, Ibaraki, 305-0003 Japan

\begin{abstract}
It is shown how a significant drawback of the electron beam induced deposition technique, namely its low deposition rate, can be circumvented. By tilting a sample a larger part of the primary electron beam energy becomes dissipated closer to the interface. This in turn increases the emission of secondary electrons, largely responsible for the deposition of the adsorbed molecule components on the surface. An order of magnitude increase in the deposition rate is reported in the fabrication of metal nanowires from organic precursor gas.
\end{abstract}

Keywords: EBID, lithography, deposition rate PACS codes: $81.16 . \mathrm{Nd}, 61.46 . \mathrm{Km}$ 


\section{Introduction}

Electron beam induced deposition (EBID) technique has been developing rapidly since first reports on metal dot and wire deposition [1, 2]. Due to the fine size of fabricated nanostructures, superior to the conventional electron beam lithography, this method is being employed in various fields (for recent reviews see $[3,4])$. The deposited nanostructures can serve either as a mask for lithographical purposes [5] or can be functionalized directly, for example, for circuitry fabrication [6]. Despite the advantages, this method is known for its low speed in comparison with the optical lithography, which is a common feature for a beam-driven patterning.

It is believed that the deposition process takes place when either the primary electron beam or generated secondary electrons decompose adsorbed precursor gas molecules on the sample surface. The dissociation cross-section for different precursor molecules depends on many experimental parameters and varies with electron energy. However, whether it is a high-energy primary electrons or lower energy $(<50 \mathrm{eV})$ secondary particles, under the normal beam incidence most of the initial energy dissipates deep in the sample and hence does not contribute to the deposition process. 
Here we show how the beam energy utilization efficiency can be improved. By tilting a sample a situation is achieved when the beam travels longer distance at the interface proximity. A beam path in the topmost layer is inversely proportional to the tilting angle cosine, drastically increasing at large angles. The inverse cosine dependence of the secondary electron yield is known to be related to this fact, since a larger number of electrons becomes generated within the electron escape depth. By applying this concept to metal nanowire deposition we show that a substantial increase in the produced nanowire length can be achieved. At the same time the nanowires deposited at grazing incidence exhibit similar profile to the ones fabricated under normal incidence geometry, indicating enhanced deposition rate as a primary cause for the observed length increase.

\section{Experimental}

To simulate beam energy distribution in the substrate Monte-Carlo simulations were performed with Casino software. For deposition experiment a Si chip with an edge polished by a set of diamond lapping films was used. The polished edge serves as a good reference for in situ actual tilt angle measurements. A scanning electron microscope (SEM, JEOL JSM-6700-F) with a precursor powder evaporation unit was employed for EBID. A $30 \mathrm{keV}$ energy beam with $30 \mathrm{pA}$ sample current $(10 \mu \mathrm{A}$ 
emission current) was used with $\mathrm{W}(\mathrm{CO})_{6}$ precursor gas at pressure $4 \cdot 10^{-4} \mathrm{~Pa}$ for all the depositions. Nanowires were characterized by high current SEM (JEOL JSM-7000F, $30 \mathrm{kV}$ beam) for length measurements (top view, normal incidence) and by scanning transmission electron microscope (STEM, JEOL JEM-2500SES) or transmission electron microscope (TEM, JEOL JEM-2100F) for cross-sectional profile observation (edge view). Samples for the edge view measurements were prepared using conventional focused ion beam (FIB) milling.

\section{Results and Discussion}

In general, secondary electrons generated in Si have energies below several tens of eV [7] and, hence, have a short stopping range within 10-20 nm [8]. In fig. 1, top, simulation results for the dissipated energy distribution in the $400 \times 400 \mathrm{~nm}$ area for the topmost $27 \mathrm{~nm}$ layer of a Si substrate are shown. It is seen how a larger fraction of the beam energy ends up in the area related to the deposition process when the sample is tilted by $87.5^{\circ}$ compared to the normal incidence case. In the same figure, below, SEM images of the actual deposited tungsten dots are shown. Their shape looks qualitatively similar to the calculated energy dissipation pattern. 
To investigate the angle effect several nanowire depositions at various angles from normal incidence up to $87.5^{\circ}$ degree tilt were performed. Each nanowire was fabricated as a linear sequence of dots with a drift compensation routine assuring sample position stability. Each dot dwell time was set at 3.3 seconds and a $2 \mathrm{~nm}$ interdot spacing for normal incidence was chosen. With sample tilt the latter value effectively increases following the inverse cosine law. On the other hand, each dot becomes more and more elongated (cf. Fig. 1) effectively forming a longer wire by the same number of dots. Two examples of thus fabricated nanowires are shown in Fig. 2, where both nanowires appear continuous and have a similar width. However, the nanowire deposited at $87.5^{\circ}$ degree tilt is longer (5670 nm vs. $880 \mathrm{~nm}$ for the normal incidence deposition wire), while consists of a less number of dots (109 vs. 371 correspondingly). Below on the same figure fabricated nanowire properties are summarized by plotting the effective wire length per dot deposited as a function of the sample tilt angle. This value was obtained by dividing measured nanowire length by the number of dots it is composed of. It is seen that the wire deposited at $87.5^{\circ}$ degree tilt is more than 20 times longer in such a representation than the one deposited at normal incidence. The inverse cosine function is also shown and the experimental points follow this curve rather precisely. Several wires with a different number of points were deposited at each angle and all of them yield the same result in normalized units. 
Nanowire cross-sectional profiles obtained by TEM characterization with FIB preparation are shown in Fig. 3. The profiles have a Gaussian shape, as would be expected for typical deposition profiles from the Monte-Carlo simulations [9]. The actual cross-section area appears to be about 2 times larger in the case of normal incidence deposition. It is important to note that the actual profile can also depend on other experimental parameters, such as beam focusing, surface condition, etc. While it is difficult to perform depositions under exactly the same experimental conditions it is nevertheless possible to assess deposition rate modification due to grazing beam incidence from the data presented. Nanowire volume $\mathrm{V}$ can be estimated for every deposition angle by multiplying the measured cross-section area by the inverse cosine factor, representing nanowire length angle dependence. Thus calculated data are shown in Fig. 4 along with the measured cross-section area values in relative units. It is seen that the net volume increase for a wire deposited at a $87.5^{\circ}$ degree tilt is about 10 times. Assuming the same composition density this increase corresponds to the change in the amount of the deposited material. The values of the deposition rate in terms of number of tungsten atoms per impinging electron can be then estimated using sample current I, deposition time per dot $\tau$, number of deposited dots $\mathrm{N}$, tungsten density $\rho_{\mathrm{W}}$ and its atomic number $\mathrm{A}_{\mathrm{W}}$ as: 


$$
\eta=\frac{\rho_{\mathrm{w}} \cdot \mathrm{V} \cdot \mathrm{e}}{\mathrm{A}_{\mathrm{w}} \cdot \mathrm{m}_{\mathrm{p}} \cdot \mathrm{I} \cdot \tau \cdot \mathrm{N}}
$$

where $\mathrm{e}$ is an elementary charge and $\mathrm{m}_{\mathrm{p}}$ is a proton mass. The actual values of the deposition rate then are $\sim 10^{-4}$ atoms $/ \mathrm{e}^{-}$for the normal incidence and $\sim 10^{-3}$ atoms $/ \mathrm{e}^{-}$for the $87.5^{\circ}$ degree tilt deposition.

In general, the inverse cosine function goes to infinity at angles approaching $90^{\circ}$. Apparently, the length of deposited wires cannot follow this function endlessly and deviation from this curve (Fig. 2) can be expected at some point. This decoupling would probably occur when there is not enough primary beam energy for adsorbed species decomposition all along the projected beam path. In other words, under these circumstances the deposition would proceed in the electron-limited regime [3]. Then, at larger deposition angles, the appearance of "broken" wires can occur and a higher linear dot density would be necessary to compensate for that effect in order to keep the wires continuous. Altogether, however, the maximum achievable deposition rate enhancement can still be higher than reported here, dependent on a particular system.

To get a better insight into the processes behind the deposition we performed experiments at various 
beam accelerating voltages. In general, dissociation cross-section $\sigma$ of the adsorbed molecules depends on the electron energy $\varepsilon$ as:

$$
\sigma \sim \frac{1}{\varepsilon} \cdot \ln \left(\frac{\varepsilon}{\varepsilon_{0}}\right)
$$

where $\varepsilon_{0}$ is a dissociation threshold energy, $\varepsilon_{0}\left(\mathrm{~W}(\mathrm{CO})_{6}\right) \approx 35 \mathrm{eV}$ [10]. This function is slowly decaying with increasing beam energy and at $30 \mathrm{kV}$ its value is about 5 times smaller than at $5 \mathrm{kV}$. Along with a better dissociation cross-section for the primary electrons a lower energy beam yields a larger number of secondary electrons. This is mainly due to smaller penetration depth and a higher probability of electron scattering in the material. Hence at lower voltages the deposition can be expected to proceed at a higher rate for the same beam current, providing the supply of precursor molecules is sufficient.

We performed deposition of wires for $5 \mathrm{kV}$ and $17 \mathrm{kV}$ beam accelerating voltage consisting of only a few points for better visualization. The overall dose, however, was the same as in the described above $30 \mathrm{kV}$ experiment. In Fig. 5 SEM images of the wires deposited at different tilt angles are shown. At both voltages with increasing angle, i.e. interdot spacing, nanowires become discontinuous and separate deposition points can be clearly discerned. However, there is a 
significant difference between these two cases in the resulting nanowire structure for larger tilt angles. While for higher voltage deposition single points remain continuous and eventually merge into a full wire at some angle (Fig. 5, right bottom), in the lower voltage case continuous wires are never observed again. Moreover, even a single point deposition yields a discontinuous structure as seen in the left bottom image.

A discontinuous structure of a single point, most likely, is a manifestation of the precursor-limited deposition regime. The number of the adsorbed gas molecules or their surface mobility is insufficient to replenish the whole area processed by the electron beam. Therefore a non-uniform coverage with deposition seed formation is observed. On the other hand, at higher beam voltages a smaller dissociation cross-section and a smaller number of secondary electrons emitted from a more confined area make the adequate supply of precursor molecules possible. It is clear that to utilize grazing incidence deposition rate enhancement effect fully a higher beam voltage should be used.

As an example of characteristic deposition times needed by EBID for conductive nanowire fabrication, it was shown that a 2 um wire can be produced in 6-7 minutes [11]. Using present 
technique this time can be shortened to at least tens of seconds per wire. Finally we note that this method can also be used for more efficient area mode depositions. An enhanced deposition rate can make feasible the fabrication of small contact pads together with nanowires by EBID technique for circuitry applications. In addition, area mode deposition can be employed for faster nanowelding, where only a local metal coating is required.

In brief, a manifold enhancement in the electron beam induced deposition rate was demonstrated on the example of tungsten nanowire fabrication. By more efficient utilization of the incident beam energy through grazing incidence an order of magnitude deposition rate enhancement was obtained. This approach can broaden the application area for the electron beam deposition technique and advance its use as a precision nanofabrication tool.

This research was partially supported by a Grant-in-Aid for Scientific Research(C) 2009-218577 from the Japan Society for the Promotion of Science, Japan, and also partially supported by the Nuclear Research Project of Ministry of Education, Science, Sports and Culture (MEXT). 


\section{REFERENCES}

[1] Koops HWP, Weiel R and Kern DP 1988 J Vac. Sci. Technol. B 6477.

[2] Kohlmann-von Platen KT, Buchmann L-M, Petzold H-C and Brunger WH 1992 J. Vac. Sci.

Technol. B 102690.

[3] van Dorp WF and Hagen CW 2008 J. Appl. Phys. 104081301.

[4] Utke I, Hoffmann P and Melngailis J 2008 J. Vac. Sci. Technol. B 261197.

[5] Guan Y, Fowlkes JD, Retterer ST, Simpson ML and Rack PD 2008 Nanotechnology 19505302.

[6] Jin CH, Wang JY, Chen Q and Peng L-M 2006 J. Phys. Chem B 1105423.

[7] Chaoui Z, Ding ZJ and Goto K 2009 Phys. Lett. A 3731679.

[8] Joy DC 1995 Monte Carlo modeling for electron microscopy and microanalysis (Oxford University Press) p.57.

[9] Mitsuishi K, Liu ZQ, Shimojo M, Han M and Furuya K 2005 Ultramicroscopy 103 17;

[10] Hagen CW, van Dorp WF, Crozier PA and Kruit P 2008 Surf. Sci. 6023212

[11] Hiroshima H, Suzuki N, Ogawa N and Komuro M 1999 Jpn. J. Appl. Phys. 387135. 


\section{FIGURE CAPTIONS}

FIG. 1 (top) Monte-Carlo simulations of the primary beam energy distribution in the 400x400 nm area of the top $27 \mathrm{~nm}$ layer for a $\mathrm{Si}$ substrate with tilted (left) and normal (right) beam incidence. (bottom) SEM images of the metal dots deposited at corresponding angles; a 400x400 $\mathrm{nm}$ area is shown.

FIG. 2 (top) SEM images of two metal nanowires deposited at different tilting angles. The wire deposited at $\alpha=0^{\circ}$ is made of 371 dots and $880 \mathrm{~nm}$ long, while the one fabricated at $\alpha=87.5^{\circ}$ consists of 109 deposition points and is $5670 \mathrm{~nm}$ long. Note that the images were taken at different magnification to accommodate the discrepancy in the nanowire length (bottom) Effective wire length per single deposited dot as a function of the incidence angle. Experimental points follow the inverse cosine law quite rigorously.

FIG. 3 Cross-sectional TEM images of the wires deposited at two different angles: $\alpha=0^{\circ}$ (top) and $\alpha=87.5^{\circ}$ (bottom). Samples were prepared by FIB milling down to $\sim 100 \mathrm{~nm}$ thick lamellas. 
FIG. 4 Nanowire cross-section area as a function of the deposition angle (dark squares); deposition rate values calculated from the measured nanowire volume (bright triangles). Inverse cosine function is also shown for reference (dotted line). A 10-fold deposition rate enhancement is most likely not an upper limit achievable by this method.

FIG. 5 SEM images of wires deposited at $5 \mathrm{kV}$ (left) and $17 \mathrm{kV}$ (right) at various tilting angles. Both wires consist of a small number of points (6-8) for better visualization of the wire formation mechanism. 


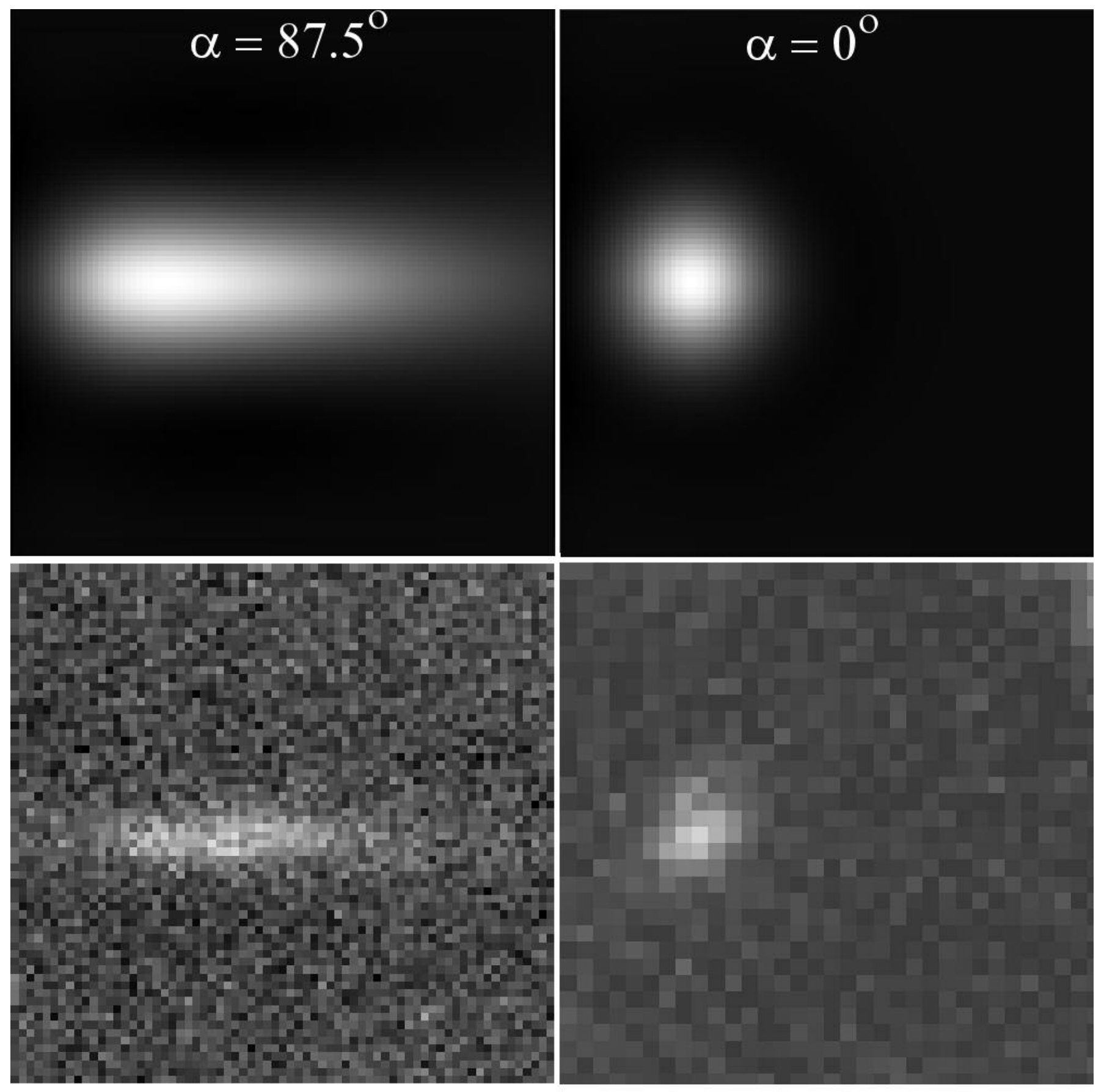

Figure 1 (Fig.1.tif) 


\section{$100 \mathrm{~nm}$}

\section{$\alpha=87.5^{\circ}$}

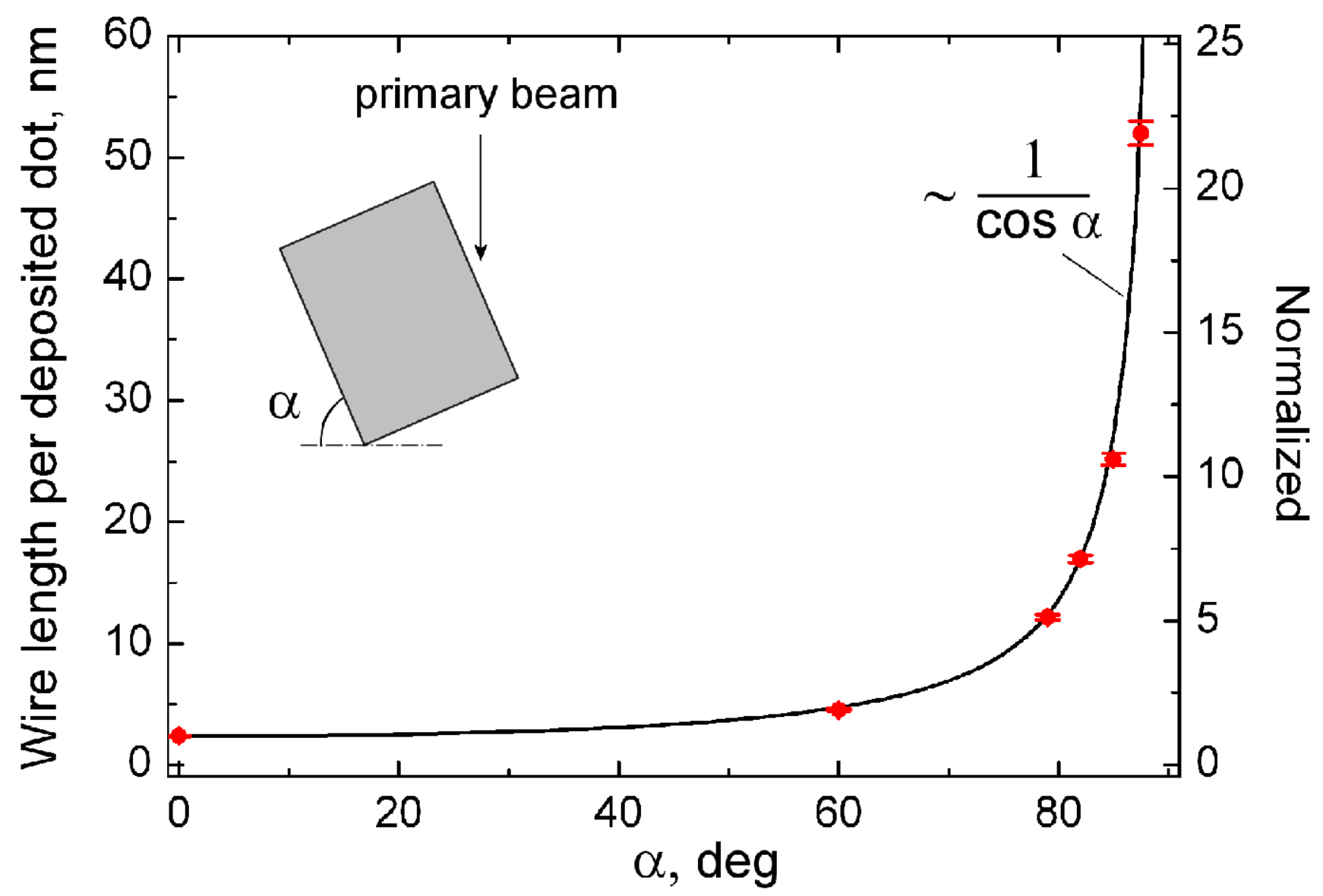

Figure 2 (Fig2.tif) 


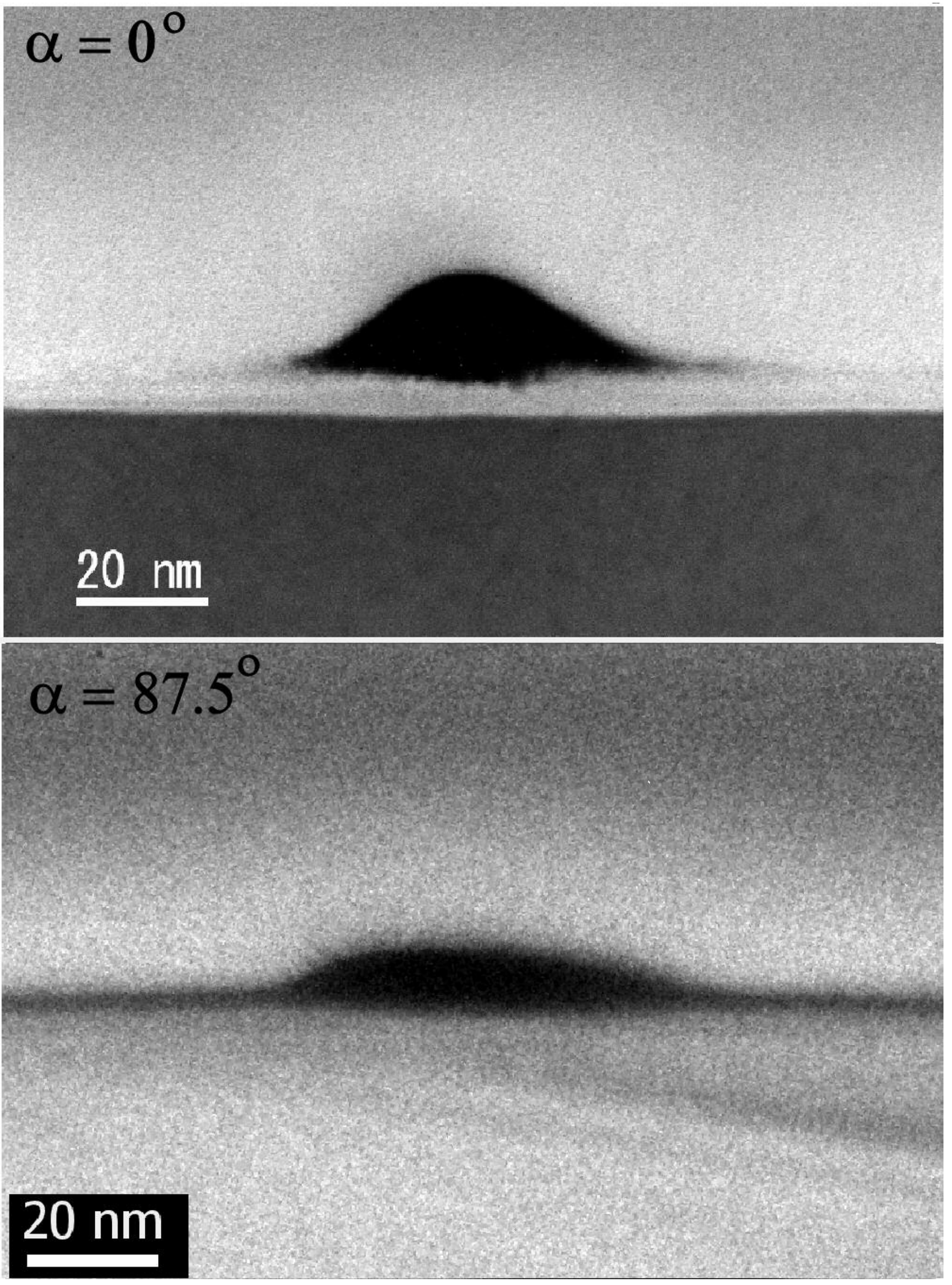

Figure 3 (Fig3.tif) 


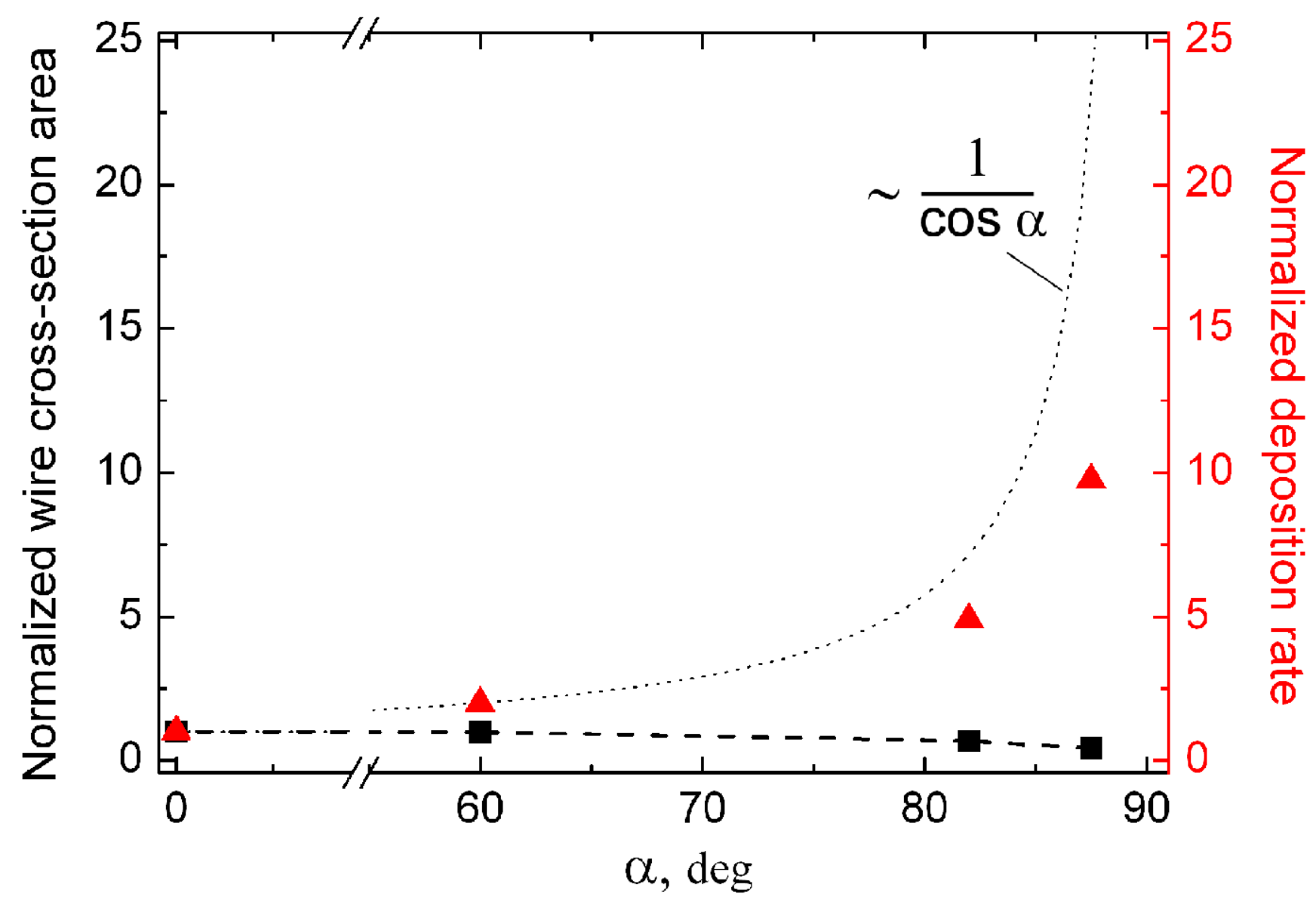

Figure 4 (Fig4.tif) 


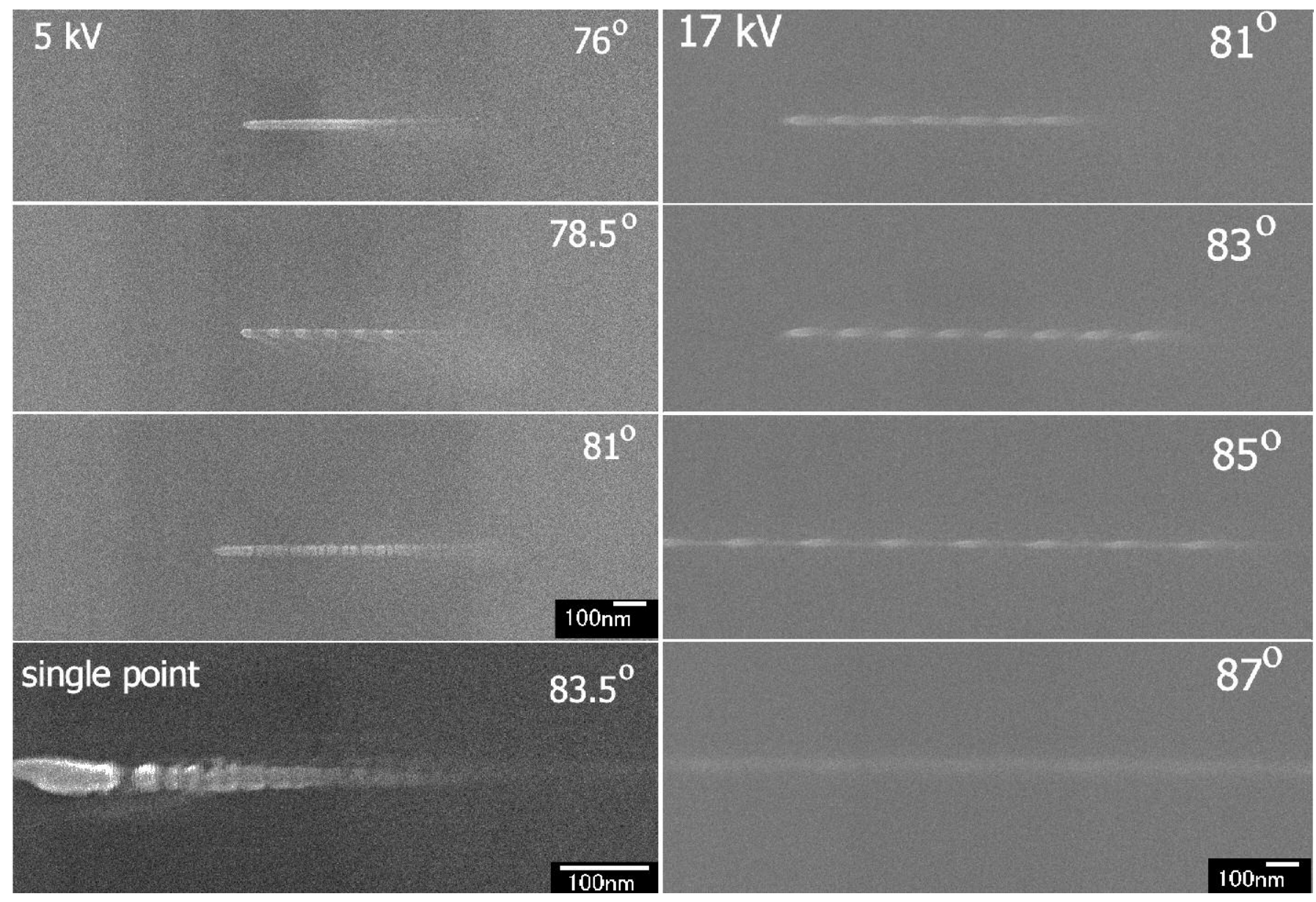

Figure 5 (Fig5.tif) 